\title{
Factores pronósticos de la bacteriemia: estudio prospectivo
}

\author{
R. PAZOS AÑÓN, R. FERNÁNDEZ RODRÍGUEZ, I. PAZ VIDAL*, \\ A. TINAJAS*, I. CANTÓN, V. ABEL, R. GONZÁLEZ, R. MARTÍNEZ, \\ P. GAYOSO**, O. FERNÁNDEZ ÁLVAREZ \\ Servicio de Medicina Interna. Enfermedades Infecciosas. *Servicio de Microbiología. \\ **Unidad de Investigación. Cristal Piñor Hospitais. CHOU. Ourense
}

\author{
PRONOSTIC FACTORS OF THE BLOODSTREAM INFECTIONS: \\ PROSPECTIVE STUDY
}

\section{RESUMEN}

Objetivo: Conocer la epidemiología e identificar los factores de riesgo que influyeron en la mortalidad de nuestros pacientes con bacteriemia.

Material y métodos: Estudio prospectivo de las bacteriemias significativas detectadas en un hospital secundario de 650 camas en el período de mayo de 1998 a mayo de 1999. La catalogación como significativa se realizó de acuerdo con el criterio del clínico y microbiólogo implicados. Se analizaron 16 variables categorizadas como clínico-epidemiológicas, factores de riesgo intrínseco y extrínseco, evolución y supervivencia. El análisis estadístico se hizo en base al programa informático SPSS; para variables cuantitativas se utilizó la media con IC del $95 \%$, y para las variables cualitativas: $\mathrm{n}^{\circ}$ y $\%$. El análisis bivariante se realizó con $t S t u$ dent y $X^{2}$; la supervivencia se expresó con gráficas de Kaplan-Meyer y el índice de regresión logística de Cox.

Resultados: Se analizaron 320 hemocultivos que tras la valoración del clínico se redujeron a 272 episodios de bacteriemia clínicamente significativa en 259 pacientes, con una incidencia de 13 casos/1000 admisiones/año. La mortalidad global fue del $22 \%$, mientras la directamente relacionada con la bacteriemia fue del $16 \%$.

La edad media fue 66,9 años (IC 95\%: 65-69) con predominio de varones $(59 \%)$, localizándose en el área médica hasta un $78 \%$ de los casos, siendo de origen nosocomial el $52 \%$ y comunitario el $48 \%$. Un $15 \%$ de los casos eran portadores de enfermedad fatal y hasta un 35\% carecían de enfermedad de base.

Según el análisis univariante se asoció con mayor de riesgo de muerte ( $\mathrm{p}<0,05)$ : la etiología (fungemias), la presencia de shock, adecuación de tratamiento, existencia de factores extrínsecos (catéter central, antibioticoterapia previa y procedimientos invasivos) y estancia hospitalaria menor de 10 días. Con el análisis multivariante sólo se identificaron como factores pronósticos independientes: Shock séptico ( $\mathrm{p}<0,0001$ con RR. 8), adecuación de tratamiento ( $<<0,001$ con $R R$ 6,67), presencia de más de 2 factores extrínsecos $(\mathrm{p}<0,04)$.

Conclusiones: La aparición de shock al diagnóstico fue la variable que más influyó en la mortalidad de nuestros pacientes, seguida de la inadecuación del tratamiento antibiótico y la existencia de factores de riesgo extrínsecos. Estas variables modificables en cierto grado obligan a incidir sobre la necesidad de un control hemodinámico agresivo y el establecimiento de un tratamiento antibiótico precoz adecuado.

\section{ABSTRACT}

Background: To identify the epidemiology and risk factors with influence in the outcome and mortality of a series of bacteriemic patients.

Material and methods: A prospective study of bloodstream infections with clinical significance detected in a secondary hospital of 650 beds over period from May 1998 to May 1999. The true bacteriemia was defined in basis to the criteria both the physician and microbiologist. A total of 16 variables were defined and categorized such as clinical-epidemiologic, intrinsic risk factor, extrinsic risk factor, outcome and survival. We used SPSS statistical package: For cuantitative variables we carried out with the mean with confidence interval of $95 \%$, for cualitative variables: number and \%. Univariate analysis of the results was carried out with the $X^{2}$ test and $\mathrm{t}$ Student, the survival was expresed with Kaplan Meyer graphics and the logistic regression model.

Results: A total of 320 positive blood cultures were studied but only 272 blood cultures were considered true bacteriemia in 259 patiens. The calculated incidence of significant episodies of bacteriemia per 1000 admissions/year was 13. The overall mortality was $22 \%$ whereas death attributable to bacteriemia was $16 \%$.

The mean age was 66.9 years (IC 95\% 65-69), 59\%episodes ocurred in men. The $78 \%$ episodes ocurred in patients hospitalized in medical services. $52 \%$ episodios were of nosocomial infection and $48 \%$ of community acquired infection. According to the severity of the underlying disease, $15 \%$ had fatl diseases and 35\% episodes ocurred in patients without underlying disease.

According to the univariant analysis, the variables which where significantly associated with greater risk death were: etilology (fungus), septic shock, the inadecuate antibiotic therapy, presence of extrinsic factors (central intravenous catheter, performance of invasive procedures, previous antimicrobial theraphy) and the hospital stay of less than 10 days. According to the multivariable analysis showed that the factors remanining independent predictors of mortality were: septic shock ( $p<0.0001$, OR: 8 ), inadecuate antimicrobial therapy ( $p<0.005$, OR: 6.7), existence of two or more extrinsic risk factors $(p<0.04)$.

Conclusions: The presence of septic shock was the most important variable which influenced in the mortality in our serie, together with inappropiate antimicrobian therapy and the asociation of various extrisinc risk factors.These variables could be modified partly, for this reason the agressive hemodinamic control and the early and appropiate antibotic therapy would be the support of the succesful bacteriemia management.

PALABRAS CLAVE: Bacteriemia. Factores pronóstico. Mortalidad.

KEY WORDS: Bacteriemia. Risk factors. Mortality.

Pazos Añón R, Fernández Rodríguez R, Paz Vidal I, Tinajas A, Cantón I, Abel V, González, R, Martínez R, Gayoso P, Fernández Álvarez O. Factores pronóstico de la bacterimia: estudio prospectivo. An Med Interna (Madrid) 2001; 18: 415-420.

Trabajo aceptado: 14 de Marzo de 2001

Correspondencia: Ricardo Fernández Rodríguez. Ruado Paseo 30, $3^{\circ}$ E post. 32003 Ourense. 


\section{INTRODUCCIÓN}

En la década de los 90 se observa un aumento en la incidencia de bacteriemias significativas junto con cambios clínicos y epidemiológicos de las mismas, todo ello condicionado por el mayor uso de materiales protésicos, accesos intravasculares, aparición de procesos patológicos (SIDA) relacionados con estados de inmunodepresión severa, y por los avances terapéuticos que condicionan técnicas intervencionistas y tratamientos antibióticos de amplio espectro. La septicemia se relaciona con una mortalidad que según las series oscila entre 17,5-40\% (1-3).

Sabemos de la existencia de factores inmodificables relacionados con la situación basal del paciente que condicionan su mortalidad global. Está descrito en la literatura que poblaciones con elevada mortalidad se relacionó ésta con la severidad de la enfermedad de base, pero este axioma se mantiene sólo en el primer mes postbacteriemia $(4,5)$.

El objetivo de este estudio se sustenta en la necesidad de conocer la descriptiva de nuestra realidad clínico-microbiológica e identificar aquellas variables independientes y potencialmente tratables que condicionan el pronóstico en la población con bacteriemia.

\section{MATERIAL Y MÉTODOS}

El Hospital Cristal-Piñor es un centro de referencia de la provincia de Ourense para enfermos agudos y crónicos con 650 camas, que carece de Servicio de Cirugía Cardíaca y Torácica; no dispone de programas de trasplante salvo de médula ósea autólogo. No se han analizado las bacteriemias detectadas en el servicio de pediatría. El $\mathrm{n}^{\circ}$ de ingresos fue de 20.653 pacientes, con una incidencia de 13 casos/1000 admisiones/año.

Se investigaron 320 hemocultivos positivos, de los cuales 50 fueron de significado incierto y 270 fueron considerados clínicamente significativos. Los datos se recogieron en el servicio de microbiología que utiliza como método de detección de hemocultivos el sistema BacT-Alert y como identificación bacteriana y determinación de la sensibilidad antimicrobiana el sistema MicroScan (Dade behrimg). Se analizaron las variables expuestas según protocolo (Tabla I) a la "cabecera del paciente", complementándose con los registros de documentación clínica hospitalaria cuando fue necesario.

\section{DEFINICIONES GENERALES}

Bacteriemia: Aislamiento de uno o varios microorganismos en hemocultivo, bien por primera vez o repetidamente dentro de los siete días siguientes al primero positivo, siempre que se tratase del mismo microorganismo y en el mismo contexto clínico del paciente.

Adquisición: Intrahospitalaria. Si se detectaba en las 72 horas posteriores al ingreso hospitalario, salvo si se relacionaban con un foco de infección detectado previamente o 5 días despues del alta.

Extrahospitalaria. Si se detectaba en las primeras 72 horas del ingreso.

\section{ENFERMEDAD DE BASE}

La gravedad se estableció en base a los criterios de McCabe y Jackson (7) englobando como fatal: la rápidamente fatal,
TABLA I

\section{VARIABLES A ESTUDIO}

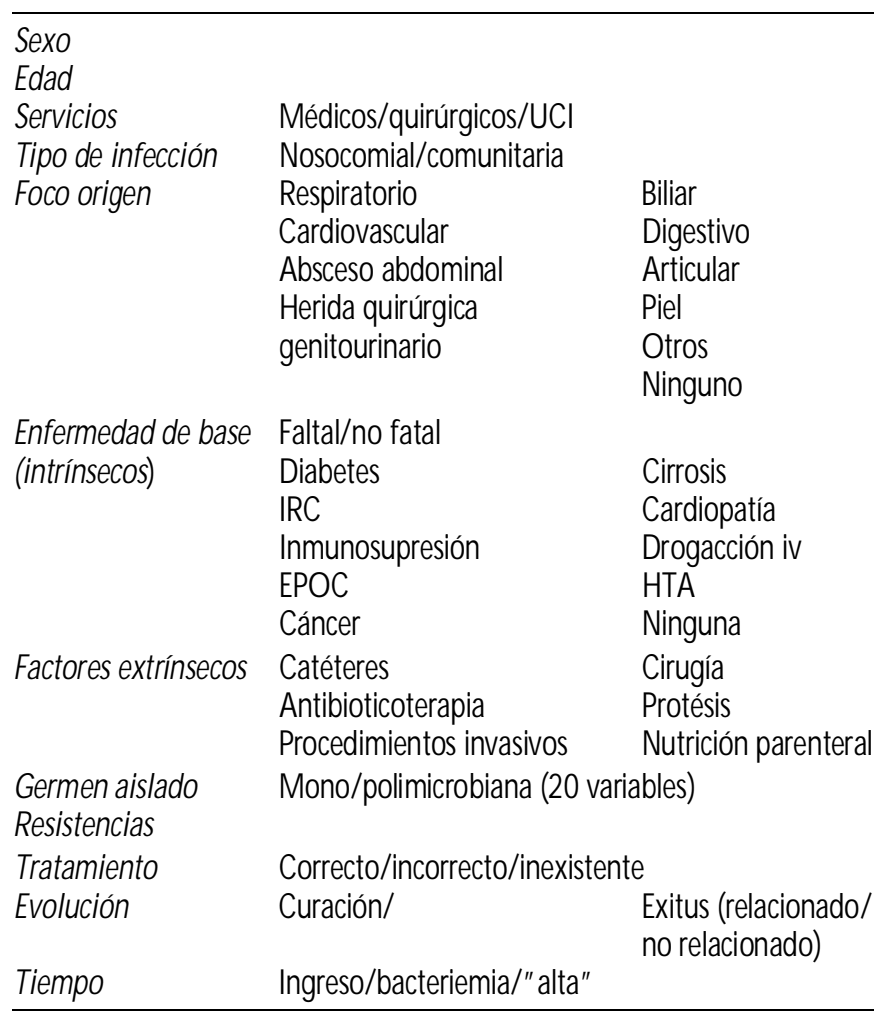

últimamente fatal y todas aquellas con una expectativa de vida inferior a 2 años, y no fatal el resto.

Se registraron como enfermedades de base: diabetes,cardiopatía, cirrosis, insuficiencia renal crónica, LCFA (limitación crónica al flujo aéreo), HTA (hipertensión arterial), cáncer, ADVP (usuarios de drogas intravenosas), inmunodepresión consecuencia de enfermedad o de tratamientos con agentes citotóxicos, corticoides o radiaciones.

Foco de origen: Se definió en función de la sospecha clínica de infección y/o del aislamiento, en dicho lugar, del mismo microorganismo. En aquellos casos que no se aisló el microorganismo en el supuesto foco, se consideró éste cuando los datos clínicos y complementarios resultaron evidentes. En función de esto se consideraron diversos focos: desconocido, respiratorio, genital, abdominal, biliar, herida quirúrgica, nefrourológico, catéteres intravenosos, osteoarticular y la existencia de 2 o mas focos posibles.

\section{FACTORES RIESGO EXTRÍNSECO}

Antibioticoterapia en la semana previa a la detección de bacteriemia.

Cirugía, en las 2 semanas anteriores a la detección del episodio.

Prótesis articular colocada en las 2 semanas previas.

Procedimientos invasivos (técnicas endoscópicas, instrumentalización respiratoria, genitourinaria, cateterización urinaria, etc) realizados en los 10 días anteriores.

Catéteres centrales colocados en la semana previa.

Administración de nutrición parenteral en la semana previa. 


\section{DATOS CLÍNICOS}

El criterio hipotensión: En pacientes previamente normotensos, se estableció cuando presentaban cifras de TA sistólica igual o inferior a $90 \mathrm{mmHg}$, acompañado de datos de mala perfusión sistémica en el momento de recogida del hemocultivo.

Se consideró fiebre cuando la temperatura axilar era mayor o igual de $38^{\circ} \mathrm{C}$.

Tratamiento antibiótico: Se consideró correcto cuando se demostró su actividad in vitro frente al o los microorganismos aislados y se administró a dosis y por vías correctas durante un período de tiempo no inferior a 7 días.

Evolución: Se distinguió entre:

Curación determinada por la ausencia de signos clínicos de infección entre 48 y 72 horas después de finalizar el tratamiento antibiótico.

Cuando el paciente falleció dentro de los 7 días posteriores al desarrollo de la septicemia por causas relacionadas con la misma, se consideró muerte directamente relacionada con la septicemia; en aquellos casos en los que la causa del fallecimiento no era evidente se consideró a la septicemia como la causa de la misma. Cuando los datos clínicos y/o microbiológicos no justificaron la causa de la muerte, se consideró que ésta no estaba directamente relacionada con la septicemia.

Resistencias: Se consideró importante identificar patrones de resistencia de los gérmenes más frecuentes con mayor traducción clínica para lo cual definimos como resistencia para: $\mathrm{S}$ aureus (oxacilina o vancomicina resistentes), S. Epidermidis (cepas vancomicina resistentes), Streptococcus pneumoniae (cepas penicilina, cefalosporinas de segunda generación o vancomicina resistentes), Enterococcus sp(cepas vancomici na o gentamicina altamente resistentes), y enterobacterias (cepas con beta lactamasas de amplio espectro )

\section{MÉTODO ESTADÍSTICO}

El análisis de los datos se ha realizado con el programa SPSS/PC para Windows. Se efectuó el estudio descriptivo de las variables estudiadas, expresando el resultado en forma de porcentaje. Para el análisis de variables cuantitativas se utilizó la media con IC del $95 \%$, y para las variables cualitativas: $\mathrm{n}^{\circ} \mathrm{y}$ $\%$. El análisis bivariante se hizo con $t$ Student; $X^{2}$, considerando asociaciones significativas aquellas con un nivel de significación de $\mathrm{p}<0,05$; la supervivencia se expresó con gráficas de Kaplan-Meyer y el índice de regresión logística de Cox.

Como indicador del riesgo se utilizó la estimación puntual de RR (riesgo relativo) con un intervalo de confianza del $95 \%$. Para el análisis multivariante se utilizó un modelo exploratorio de regresión logística, en el que la variable dependiente fue la mortalidad relacionada de los enfermos bacteriémicos. El análisis se ha realizado con el procedimiento Logistic Regretion del paquete estadístico SPSS/PC versión para Windows.

\section{RESULTADOS}

\section{EPIDEMIOLOGÍA}

Se detectaron 272 episodios de bacteriemia significativa en 259 pacientes con una incidencia de 13 casos /1000 admisiones. La mortalidad total fue del $22 \%$, mientras que la directamente atribuida a la bacteriemia fue del $16 \%$. La edad media era de 66,7 años (IC95\% 65-69), con predominio de sexo masculino $(59,4 \%)$, aunque las diferencias no fueron significativas

En el área médica se concentraba el $78 \%$ de los aislamientos,siendo un $52 \%$ de adquisición intrahospitalaria. Hubo diferencias en la mortalidad, $16 \%$ para las nosocomiales y un $13 \%$ para las comunitarias, sin significación estadística.

Un $15 \%$ de los pacientes presentaron shock al diagnóstico,su presencia se confirmó como factor de riesgo independiente de mortalidad $(\mathrm{p}<0,0001)$ asociándose a un riesgo relativo de muerte de 8 (Figura 1). La ausencia de fiebre en nuestro estudio no se relacionó con aumento de mortalidad.

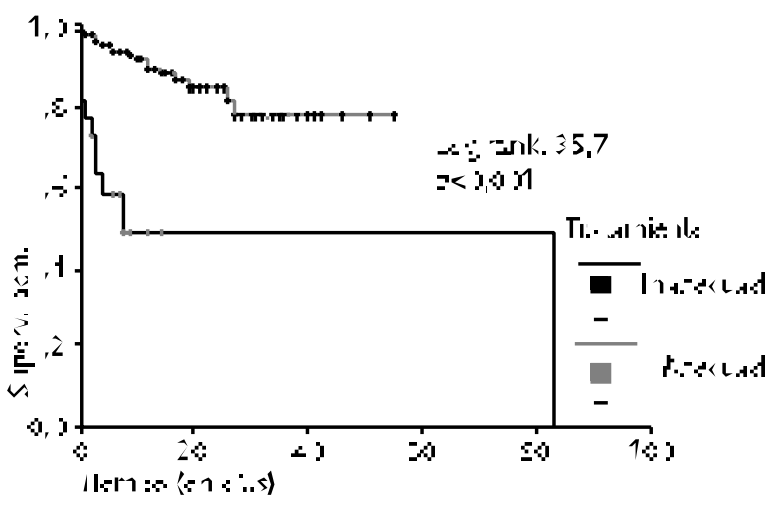

Fig. 1. Función de supervivencia según adecuación del tratamiento.

Enfermedad de base: $15 \%$ eran portadores de una enfermedad de base fatal, un $50 \%$ enfermedad no fatal y hasta un $35 \%$ carecían de enfermedad de base. El $33 \%$ de los pacientes con enfermedad de base eran portadores de neoplasia y/o inmunodepresión. En este grupo el riesgo de muerte fue mayor pero no en términos de significación estadística.

El porcentaje de mortalidad relacionada con bacteriemia es análoga tanto para enfermedad fatal como no fatal(16\% vs $17 \%$ ), mientras que la mortalidad global es mayor para la enfermedad fatal.

Foco de origen: Un $26 \%$ correspondía a cardiovascular; incluyendo endocarditis e infección de catéteres (periféricos y/o centrales), documentados clínica o microbiológicamente.En segundo lugar el foco genitourinario (18\%), respiratorio (14\%), biliar $6 \%$, abscesos abdominales $6 \%$ y hasta un $12 \%$ el foco fue desconocido En este último grupo predominaba el origen nosocomial.

Factores extrínsecos: En el $47 \%$ de los aislamientos no se identificaron factores predisponentes, un $31 \%$ eran portadores de un único factor de riesgo, y hasta un $22 \%$ tenían 2 o más factores predisponentes.

En los que se identificó factor predisponente había un claro predominio intrahospitalario (72\%) siendo hasta un $24 \%$ portadores de catéter intravascular central, un $21 \%$ habían sido sometidos a procedimientos invasivos y un $17 \%$ a antibioticoterapia en días previos.

\section{ESTUDIOS MICROBIOLÓGICOS}

$55 \%$ de los aislamientos eran Gram (+), un 39\% eran Gram (-), y un $3 \%$ correspondía a anaerobios y un $3 \%$ a levaduras. Los 
principales aislamientos fueron $\mathrm{E}$ coli $23,7 \%$, S coagulasa negativos $23 \%, \mathrm{~S}$ aureus $15,6 \%$, Streptococcus pneumoniae $6,3 \%$ (Figura 2). No se identificaron diferencias de mortalidad en cuanto al tipo de germen salvo para levaduras $(\mathrm{p}<0,048)$.

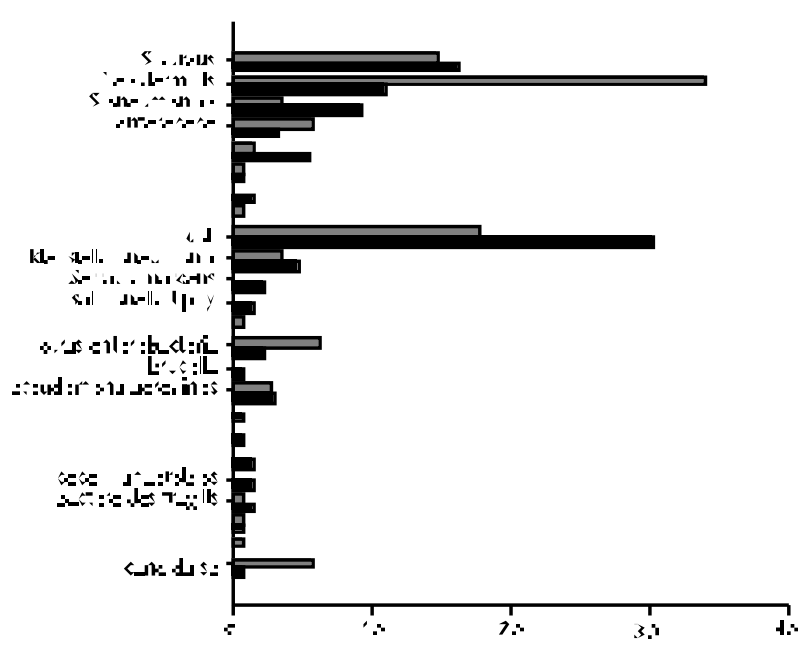

Fig. 2. Distribución de germenes según tipo de infección.

Un $12 \%$ de los aislamientos eran polimicrobianos asociándose en un $60 \%$ a la presencia de catéteres, antibioticoterapia previa y nutrición parenteral, siendo los focos más habituales el cardiovascular (25\%), genitourinario (22\%) y digestivo $(12,5 \%)$. No se objetivó un aumento de la mortalidad significativa en este grupo.

\section{TRATAMIENTO}

Un $9 \%$ de los pacientes no recibió tratamiento o este fue inadecuado, suponiendo en el área quirúrgica hasta un $18 \%$. La adecuación del tratamiento se reveló como un factor pronóstico independiente de muerte; con un riesgo relativo de 6 $(\mathrm{p}<0,001)$

En cuanto a la estancia hospitalaria se ha detectado el mayor riesgo de muerte en los 10 días posteriores al episodio.

\section{RESISTENCIA}

Este estudio no fue diseñado para determinar la prevalencia de resistencia antibiótica, aunque por la repercusión clínica que puede representar el aislamiento de determinadas cepas realizamos el análisis de los patrones de resistencia de los gérmenes más frecuentes. No se identificó ninguna cepa de Streptococcus pneumoniae, aislada en hemocultivos, resistente a penicilina o cefalosporinas de tercera generación. Un $12 \%$ de los $\mathrm{S}$ aureus fueron meticilín resistentes (MARSA), sin documentarse cepas resistentes a vancomicina. En el periodo de estudio no se detectó ningún brote de MARSA en nuestro hospital. Ningún caso de $S$ coagulasa negativo fue resistente a la vancomicina. Un $33 \%$ de las cepas de Enterococo fueron resistentes a la ampicilina y/o gentamicina, aunque no se detectó ninguna especie de alta resistencia a la gentamicina o a la vancomicina. Para E coli se encontraron un $26 \%$ de resistencias a la ampicilina .

\section{DISCUSIÓN}

La alta tecnificación de la medicina actual que conlleva la implantación de materiales protésicos y el uso de accesos intravasculares, la aparición de nuevas enfermedades como el SIDA y los avances en el campo del tratamiento definidos por el uso de terapias altamente "agresivas" y el empleo de antibioticoterapia de amplio espectro condicina cambios en la flora habitual intra y extrahospitalaria $(1,2,8,9)$.

Un hecho muy relevante en nuestro medio es el envejecimiento de la población que obliga al ingreso en instituciones cerradas de un importante porcentaje de población; por otro lado, se está tendiendo al tratamiento ambulatorio de patologías tradicionalmente "hospitalarias", corroborado en la expansión de los "hospitales de día". Desde esta perspectiva parece un momento oportuno para redefinir el criterio de infección nosocomial, como ya se ha puesto de manifiesto en las últimas directrices de los Center Disease Control $(10,11,23)$.

Del mismo modo que nos situamos en el contexto poblacional, tampoco podemos sustraernos de las características propias de nuestro medio hospitalario, un hospital de nivel secundario, de modo que los resultados de mortalidad global en torno al $22 \%$, y la incidencia de 13 casos por mil admisiones son concordantes con otras series de nuestro medio $(12,13)$.

Se sabe que la presencia de bacteriemia incrementa el riesgo de muerte en un paciente hasta 14 veces $(12,14)$, siendo la edad avanzada una variable de mal pronóstico presente en todas las series consultadas $(1,3,12,15,16)$. El hecho de que no ocurra en nuestra serie puede venir determinado por la homogeneidad de la población a estudio,anciana con edad en torno a 70 años, excluída pediatría del diseño inicial. Asimismo la edad media de muerte para los pacientes con bacteriemia se encuentra en los 70 años, mientras que para los que presentaban muerte no relacionada se situa en los 66 años, superponiéndose ambos intervalos de confianza (Figura 3).

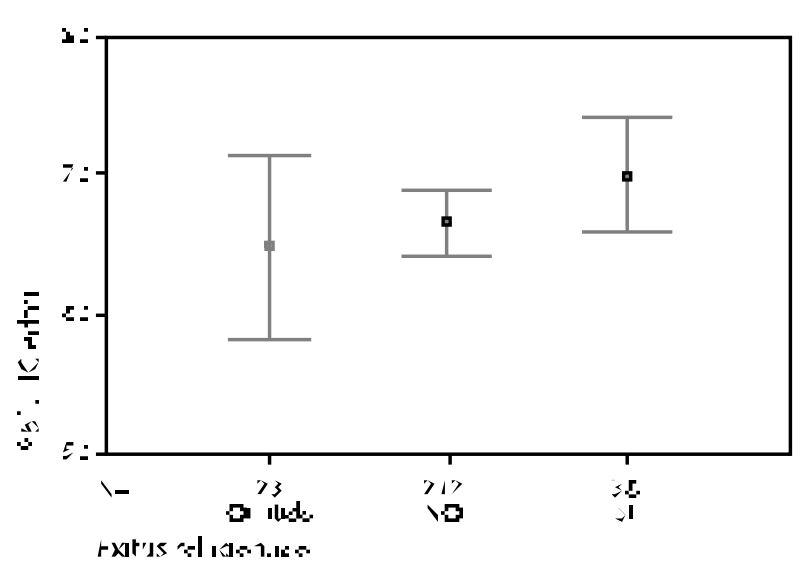

Fig. 3. Edad según evolución clínica.

El foco cardiovascular fue el más frecuente en nuestro medio, lo cual predispone a una bacteriemia por microorganismos de la flora cutánea sobre todo SCN (23\%) y S. aureus (15\%).

En las revisiones más recientes de otras series $(1,11,15)$, se observa el creciente papel de los $S$. coagulasa negativos como patógenos lo que obliga a una valoración minuciosa de los presumibles contaminantes. 
Es un hecho bien conocido que la clínica rara vez ayuda, así las flebitis asociadas a catéteres periféricos son habitualmente expresión de fenómenos inflamatorios no infecciosos producidos por las soluciones y fármacos administrados y los catéteres centrales rara vez se asocian a inflamación cuando se infectan, por lo cual el criterio clínico aplicado en nuestra definición podría ser responsable de sobreestimación de catéteres infectados. Parece necesario la generalización y estandarización de las técnicas cuantitativas o semicuantitativas de procesamiento de catéteres y la necesidad de adoptar criterios estrictos de infección versus colonización de catéter en nuestro medio $(16,17,18,19)$.

Se confirma en nuestra serie el discreto predominio de los Gram (+) (55\%) sobre los Gram (-) relacionado con maniobras terapéuticas que implican la colocación de cuerpos extraños, aparición de nuevos mecanismos de resistencia y mejor tratamiento de las bacteriemias de los Gram (-) (8).

Dentro de las fungemias, Cándida sp es el agente etiológico más frecuente ;al mismo tiempo se observa un incremento de las especies no albicans que en algunas publicaciones supone hasta el $50 \%$, lo que puede ser debido a la amplia difusión de medicamentos antifúngicos, especialmente imidazoles para el tratamiento y como profilaxis $(12,20,21,22)$. En nuestro estudio la mortalidad es superior (mortalidad relacionada 40\%)con respecto al resto de grupos de microorganismos.

No detectamos diferencias significativas en cuanto a la mortalidad en pacientes bacteriémicos según su enfermedad de base (fatal/no fatal)que fueron muy similares (17\% vs $16 \%$ ), mientras que la mortalidad global fue mayor para los pacientes portadores de enfermedad fatal. Parece que pudieran existir otros factores independientes de la situación basal del paciente que condicionan la mortalidad del proceso infeccioso. Este hecho fue puesto de manifiesto por el grupo de MD Rojo (12) recientemente, aunque los estudios previos de la literatura no lo avalan $(3,6,7)$.

En general se considera que el origen nosocomial de las infecciones es un factor de mal pronóstico, sin embargo en nuestra experiencia la mortalidad fue muy pareja $(15,9 \%$ frente $13,2 \%$ en comunitaria), conclusiones semejantes a las de otro estudio de nuestro medio (12).

Entre los factores de riesgo extrínseco, la presencia de catéter venoso central, la antibioticoterapia previa y la realización de procedimientos invasivos en días previos se ha asociado con un índice de letalidad de las bacteriemias significativamente más alto que en presencia de otros factores predisponentes o en ausencia de los mismos, aunque no se pueden considerar variables independientes; sólo la presencia de 2 o más factores de riesgo frente a la ausencia de los mismos condiciona una mortalidad mayor estadísticamente significativa $(\mathrm{p}<0,04)$. El mayor riesgo de muerte asociado con determinados factores predisponentes se debe al gran número de manipulaciones y procedimientos diagnósticos o terapéuticos a que se ven sometidos ciertos pacientes.

El análisis del tratamiento antibiótico revela algo tan esperado como que la existencia del mismo, independientemente del tipo que sea, mejora significativamente el pronóstico $(1,5,6,12,15)$. En nuestra serie los mal tratados o no tratados oscilan entre un $10 \%$ ( área médica) y un $18 \%$ en el área quirúrgica. El riesgo relativo de mortalidad para tratamiento inadecuado en nuestra serie es de 6, ( $p<0,001)$ (Figura 4).

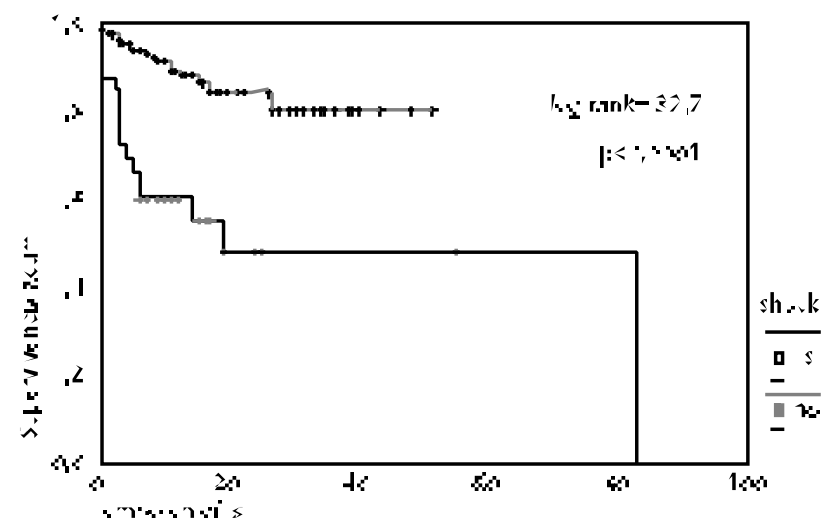

Fig. 4. Funciones de supervivencia según situación hemodinámica.

De igual forma, la presencia de shock se ha constatado en nuestra serie como en la mayoría de la literatura $(1,4,6,12,13,15)$ como uno de los factores que más ha influido en la mortalidad de la bacteriemia, independientemente de la edad, patología de base y otros factores que pudiesen actuar como confusores, con un riesgo relativo de $8(\mathrm{p}<0,0001)$

Al analizar el tiempo de supervivencia desde la bacteriemia documentada, hemos objetivado que el mayor riesgo de muerte se produce en los primeros 10 días , lo que corrobora la idea clásica que las complicaciones directas de la bacteriemia son de carácter precoz $(4,5)$.

La aparición de shock al diagnóstico fue la variable que más influyó en la supervivencia en nuestra serie, seguida de la inadecuación del tratamiento y la existencia de factores de riesgo extrínseco; todos ellos en principio potencialmente MODIFICABLES. Por lo tanto, independientemente de la situación de base del paciente, parece necesario establecer un tratamiento antibiótico empírico precoz e insistir en un control hemodinámico agresivo al diagnóstico.

\section{Bibliografía}

1. Weinstein MP, Towns ML, Quartey SM, Mirrett S, Reimer LG, Parmigiani $\mathrm{G}$ et al. The clinical significance of positive blood cultures in the 1990s: A prospective comprehensive evaluation of the microbiology, epidemiology and outcome of bacteriemia and fungemia in adults. Clin Infect Dis 1997; 24: 584-602

2. Weinstein MP , Reller LB, Murphy JR, Lichtenstein KA. The clinical significance of positive blood cultures: a comprehensive analysis of 500 episodes of bacteremia and fungemia in adults. Laboratoriy and epidemiologic observations. Rev Infect Dis 1983; 5: 35.

3. Miller PJ, Wenzel RP. Etiologic organisms as independent predictors of death and morbidity associated with bloodstream infections. J Infect Dis 1987; 156: 471-77

4. Bates David W,Pruess Kim E, Lee Thomas H; MD;MSc. How bad are bacteremia and sepsis? Outcomes in a cohort with suspected bacteremia.Arch Intern Med 1995; VOL 155: 593-598.

5. Aube H,Milan C, Blettery B. Risk factors for septic shock in the early agement of bacteremia. Am J Med 1992; 93: 283-288.

6. Grupo de estudio de bacteriemia. Bacteriemia en seis hospitales españoles 
Med Clin (Barc) 1986; 86: 221-232.

7. Mc Cabe WR, Jackson GG. Gram negative bacteremia. Etiology and ecology. Arch Intern Med 1962; 110: 847-855.

8. Bouza E, Pérez- Molina J, Muñoz P. Report of ESGNI-001 and ESGNI002 studies. Bloodstream infections in Europe.Clin Microbiol Infect 1999; 5: 2S1-2S2.

9. Cockerill Fr, Hughes JG, Vetter EA, Mueller RA,Weaver AL, Ilstrup DM, et al. Analysis of 281.797 consecutive blood cultures performed over an eight year period:trends in microorganisms isolated and the value of anaerobic culture of blood. Clin Infect Dis 1997; 24: 403-18.

10. Moran T, White J, Jarvis W, Emori TG, Culver DH, Munn VP et al. Nosocomial infection surveillance. MMWR 1984; 35: 1SS, 17 SS29SS.

11. Ezpeleta Baquedano C, Sota Busselo M, et al. Bacteriemia: estudio multicéntrico español con 5000 casos. Rev Clin Esp 1997; 197: 15-20

12. Rojo MD, Pinedo A, Clavijo E. Factores que influyen en la evolución de la bacteriemia: Estudio prospectivo de un hospital universitario. Enferm Infecc Microbiol Clín 1999; 17: 439-444.

13. Matas L, Marti C, Morera MA. Bacteriemia en 13 hospitales generales de la provincia de Barcelona. Estudio prospectivo de 1674 episodios. Enferm Infecc Microbiol Clin 1995; 13: 345-55

14. Bouza E. Monografías clínicas en enfermedades infecciosas: Bacteriemia y septicemia. Barcelona. Ediciones Doyma 1991.
15. Haug JB, Harthug S, Kalager R. Blodstream infections at a norweian university hospital 1974-1979 and 1988-1989: Changing etiology, clinical features and outcome. Clin infect Dis 1994; 19: 246-256

16. Villar A, Blanes A, Sánchez J. Estudio multifactorial pronóstico de la sepsis en un servicio de Medicina Interna. An Med Interna (Madrid) 1993; 10: 107-112.

17. Navas E. Bacteriemia asociada a catéter. Monográfico de bacteriemia. Rev Clin Esp 1997: 5; 41-44.

18. Pearson ML. Guidelines for prevention of intravascular device-related infections. Am J Infect Control 1996; 24: 262-293

19. Proyecto EPINE 1992. Sociedad española de Higiene y Medicina Preventiva Hospitalrias y grupo de trabajo EPINCAT.

20. Yamamura Dl, Rotstein C, Nicolle LE. Candidemia at selected Canadian sites: results from the Fungal Disease Registry 1992-1994. CMAJ 1993; 160: 493-9

21. De Marie S. New developments in the diagnosis and management of invasive fungal infections. Haematologica 2000; 85: 88-93.

22. Debusk-CH, Daoud-R, Thirumoorthi MC. Candidemia:current epidemiologic characteristics and a long-term follow.up of the survivors. Scand J Infect Dis 1994; 26: 697-703

23. Yokoe Ds, Anderson J, Chambers R, Connor M, Finberg R, Hopkins C, Lichtenberg D, Marino S, et al. Simplified surveillance for nosocomial bloodstream infection. Infect Control Hosp Epidemiol 1988; 19 (9): 657-60. 